\title{
Identificação de emoções e sentimentos: estudo exploratório com alunos do ensino básico
}

Identifying emotions and feelings: exploratory study with elementary and high school students Identificación de emociones y sentimientos: estudio exploratorio con alumnos de educación primaria

Graça Aparício io https:// orcid.org/0000-0001-6149-062X

Manuela Ferreira iD https:// orcid.org/0000-0002-8452-2222

Sofia Campos iD https://orcid.org/0000-0002-4696-3537

Olivério Ribeiro io https://orcid.org/0000-0001-7396-639x

Odete Amaral id https://orcid.org/0000-0002-3382-6074

Carla Cruz io https://orcid.org/0000-0002-7897-0424

João Duarte id https://orcid.org/0000-0001-7082-8012'

Rosa Martins io https://orcid.org/0000-0001-9850-98221

Como citar:

Aparício G, Ferreira M, Campos S, Ribeiro

0 , Amaral 0 , Cruz C, et al. Identificação de

emoções e sentimentos: estudo exploratório

com alunos do ensino básico. Acta Paul Enferm. 2020;33:e-APE20190057

DOI

http://dx.doi.org/10.37689/ acta-ape/2020A00057

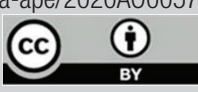

Descritores

Emoç̃oes; Criança; Adolescente; Instituições acadêmicas; Estudantes; Saúde mental

Keywords

Emotions; Child; Adolescent; Schools; Students;

Mental health

Descriptores

Emociones; Niño; Adolescente; Instituições académicas; Estudiantes; Salud mental

Submetido

9 de Março de 2019

Aceito

30 de Setembro de 2019

\section{Autor correspondente}

Carla Cruz

E-mail: cruzcarla@hotmail.com

\section{Resumo}

Objetivo: Avaliar a competência de alunos do $2^{\circ}$ e $3^{\circ}$ ciclo do Ensino Básico na identificação e diferenciação de emoções e sentimentos.

Métodos: Estudo exploratório de corte transversal, desenvolvido no âmbito do Projeto MaiSaúdeMental com referência CENTR0-01-0145-FEDER-023293, desenvolvido na Escola Superior de Saúde do Instituto Politécnico de Viseu, Portugal. Foi realizado com uma amostra de conveniência constituída por 101 crianças/ adolescentes. Foi utilizado um questionário de caracterização sociodemográfica e o Inventário de Identificação de Emoções e Sentimentos. Este é um inventário de auto-relato que avalia a capacidade das crianças/ adolescentes identificarem e diferenciarem emoções e sentimentos, partindo de situações quotidianas. É composto por 15 itens que correspondem a 15 situações. A aplicação do questionário decorreu em sala de aula, após uma breve explicação do seu funcionamento e esclarecimento de dúvidas. No tratamento dos dados foi utilizado o programa informático Statistical Package for the Social Sciences ${ }^{\circledR}$ (SPSS versão 24.0) e efetuada análise descritiva e analítica, considerando-se para análise dos testes o Intervalo de Confiança (IC) a 95\% (significância $p<0,05$ ).

Resultados: Os alunos tinham entre 9 e 15 anos de idade e a maioria $(27,7 \%)$ frequentava $08^{\circ}$ ano de escolaridade. Globalmente identificam poucas emoções e sentimentos, numa média de 13,36 pontos para um score máximo de identificação emocional de 39, sendo que em média identificam mais emoções e sentimentos positivos (5,11 pontos) do que negativos (4,33 pontos) e neutros (3,91 pontos). Para todas as dimensões os valores médios são mais elevados no sexo feminino e nos alunos de 14 anos, mas apenas significativos em algumas das diferenciações emocionais $(p<0,05)$.

Conclusão: Os resultados encontrados vão ao encontro de investigações nesta área, concluindo-se que as crianças e adolescentes apresentaram fraca capacidade de identificação e diferenciação de emoções, evidenciando contudo maior capacidade de diferenciação emocional positiva do que negativa e/ou neutra. Verificou-se ainda que existem maiores competências de diferenciação e identificação emocional no género feminino e que essas competências aumentam com a idade.

\section{Abstract}

Objective: To assess the competence of students from Grade 5 to 10 of elementary and high school to identify and differentiate emotions and feelings.

Methods: This was cross-sectional, exploratory study, carried out during the Project "MaiSaúdeMental" (More Mental Health), at the referall CENTR0-01-0145-FEDER-023293, and developed in Escola Superior de Saúde of Instituto Politécnico de Viseu, Portugal. This was a convenience sample including 101 children/adolescents. 
A questionnaire for sociodemographic characterization and an Inventory to Identify Emotions and Feelings were used. This self-reporting inventory evaluated the capacity of children/adolescents to identify and differentiate their emotions and feelings, based on everyday situations. The questionnaire was composed of 15 items that correspond to 15 situations. The questionnaire was applied in the classroom, after a short explanation on how it worked and clarification to questions of participants. Statistical Package for the Social Sciences ${ }^{\circledR}$ (SPSS version 24.0) was used for data process, and a descriptive and analytical assessment was performed. The 95\% confidence interval for the tests analysis was considered significant $(p<0.05)$.

Results: The students were aged between 9 and 15 years old, and the majority (27.7\%) was attending grade 8. In general, students identified few emotions and feelings, a mean score of 13.36 points for a maximum score of 39 of emotion identification, whereas on average they identify more positive emotions and feelings (5.11 points) than negative (4.33 points) or neutral (3.91 points). In all dimensions, mean values were higher in female students and in 14-year-0ld students, but slightly significant for some emotion differentiations $(p<0.05)$.

Conclusion: The results found corroborate with investigations in this area. Children and adolescents have poor capacity to identify and differentiate emotions, although they show greater capacity to differentiate positive emotions rather than negative or neutral emotions. We also observed that female students have more competence to differentiate and identify emotions, and these competences increase with age.

\section{Resumen}

Objetivo: Evaluar la competencia de alumnos de $5^{\circ}$ a $9^{\circ}$ año de primaria en la identificación y diferenciación de emociones y sentimientos.

Métodos: Estudio exploratorio de corte transversal, desarrollado en el ámbito del Proyecto "MaiSaúdeMental" (Más Salud Mental), referencia CENTR0-010145-FEDER-023293, llevado a cabo en la Escola Superior de Saúde del Instituto Politécnico de Viseu, Portugal. Se realizó con una muestra de conveniencia formada por 101 niños/adolescentes. Se utilizó un cuestionario de caracterización sociodemográfica y el Inventario de Identificación de Emociones y Sentimientos. Es un inventario de autorrelato que evalúa la capacidad de los niños/adolescentes de identificar y diferenciar emociones y sentimientos, a partir de situaciones cotidianas. Se compone de 15 ítems que corresponden a 15 situaciones. La aplicación del cuestionario se realizó en el aula, luego de una breve explicación de su funcionamiento y esclarecimiento de dudas. Para el tratamiento de datos, se utilizó el programa informático Statistical Package for the Social Sciences ${ }^{\circledR}$ (SPSS versión 24.0) y se realizó un análisis descriptivo y analítico, en el que se consideraron para el análisis de las pruebas el intervalo de confianza (IC) a 95\% (significación $p<0,05)$.

Resultados: Los alumnos tenían entre 9 y 15 años de edad y la mayoría (27,7\%) estaba en $8^{\circ}$ año de escuela. De forma global, identifican pocas emociones y sentimientos, un promedio de 13,36 puntos en una puntuación máxima de identificación emocional de 39, de modo que, en promedio, identifican más emociones y sentimientos positivos (5,11 puntos) que negativos (4,33 puntos) y neutros (3,91 puntos). En todas las dimensiones, los valores promedio son mayores en el sexo femenino y en alumnos de 14 años, pero poco significativos en algunas diferenciaciones emocionales $(p<0,05)$.

Conclusión: Los resultados encontrados están en línea con las investigaciones de esta área, con lo que se concluye que los niños y adolescentes presentan poca capacidad de identificación y diferenciación de emociones, sin embargo se observa mayor capacidad de diferenciación emocional positiva que negativa o neutra. Además, se verificó que existen mayores competencias de diferenciación e identificación emocional en el género femenino y que esas competencias aumentan con la edad.

\section{Introdução}

A Competência emocional pode ser definida como uma ferramenta que permite dar resposta às exigências do contexto social, ajudando a criança e o adolescente a lidar com os desafios nos vários ambientes em que interagem, permitindo ainda reconhecer como a comunicação das emoçóes e sentimentos afetam as relaçóes. ${ }^{(1,2)}$ Esta competência inclui, para além de outras, ser capaz de reconhecer o estado emocional ou a possibilidade de experienciar múltiplas emoçóes, de identificar e diferenciar as emoçôes dos outros e utilizar vocabulário e expressão de emoçôes e sentimentos de acordo com o seu nível de desenvolvimento, o que inclui a capacidade de adaptação e gestão de emoçóes menos positivas. Alguns estudos ${ }^{(2-4)}$ têm demonstrado que os indivíduos com alta diferenciação emocional são mais precisos no reconhecimento das expressóes faciais emocionais dos outros, sugerindo que o conheci- mento que usamos para entender a nossa própria experiência emocional, nos ajuda também a entender as emoçóes daqueles com que nos relacionamos. A diferenciação emocional pode assim ser entendida como a capacidade de usar palavras de emoção adequadas à especificidade das situaçóes emocionais sentidas no momento e não de forma indiferenciada. Contudo a diferenciação emocional não se refere à riqueza do vocabulário que expressa a emoção em si, mas ao uso adequado e diferenciado de palavras, direcionadas para situações emocionais específicas. ${ }^{(3,4)} \mathrm{A}$ importância da diferenciação emocional no bem-estar subjetivo tem sido demonstrada em vários estudos ${ }^{(4,5)}$ que sugerem uma relação negativa entre diferenciação emocional e psicopatologia. Por exemplo, uma maior diferenciação emocional está relacionada com uma melhor capacidade de regulação emocional, melhor auto-estima, menor intensidade de emoçóes negativas sentidas, menor stresse, menos neuroticismo e depressão e maior 
empatia, o que, segundo alguns estudos se associa a ligaçôes afetivas mais estáveis. ${ }^{(4-7)}$ Pelo contrário, a incapacidade de diferenciar emoçóes pode promover comportamentos desadaptativos em resposta a uma emoção intensa, tal como a raiva e outros comportamentos agressivos associados a problemas do comportamento. ${ }^{(5,6)}$

No âmbito da aprendizagem e da expressão de emoções, evidências apontam para diferenças, ainda que nem sempre consistentes, relacionadas com o gênero. Numa revisão sistemática com mete-análise de estudos com crianças e adolescentes, integrando 166 estudos, num total de 21.709 participantes apurou-se, no global, diferenças significativas pequenas, apresentando as meninas mais emoçóes positivas e emoçóes internalizantes (ie: tristeza, ansiedade, simpatia) que os meninos. Estas diferenças foram mais pronunciadas com o aumento da idade, revelando os meninos mais emoçóes externalizantes que as meninas na idade pré-escolar e menos na adolescência. ${ }^{\left({ }^{8}\right)}$

Face a estas evidências, torna-se clara a importância da promoção de competências emocionais, dado o seu impacto no sucesso relacional e social das crianças e jovens nos vários ambientes em que se integram e em especial na família e na escola, dada a sua importância enquanto intervençóes preventivas em saúde mental. Estas intervençôes devem dirigirse não só à promoção de competências emocionais nas crianças, mas aos pais e professores para que consigam, de forma mais eficaz, auxiliar as crianças a nomear, diferenciar e regular as suas emoçóes e compreender as emoções dos outros, a favor de um mais equilibrado e ajustado funcionamento intra e interpessoal. Um estudo com grupo controle, ${ }^{(9)}$ realizado numa amostra de 628 alunos do $7^{\circ}$ ao $9^{\circ}$ ano de escolaridade, apurou que após a o programa de intervenção "Atitudes Positivas", as crianças do grupo em estudo apresentavam ganhos significativos em três das cinco competências emocionais, especificamente na consciência social e autocontrole, bem como diminuição dos níveis de ansiedade. Os resultados evidenciaram ainda que o sexo feminino revelou maiores ganhos na consciência social e maiores reduçôes nos níveis de isolamento e ansiedade social, quando comparados ao sexo oposto. Os alunos do grupo de intervenção, com menores índices obtidos no pré-teste, no âmbito da consciência social, lucraram mais do que os que integravam o grupo controle. Estes resultados permitiram inferir que a intervenção melhorou as competências sociais e emocionais dos alunos do ensino médio, apoiando a generalização transcultural da eficácia dos programas de aprendizagem socioemocional. O desenvolvimento destas competências, através da implementação de programas de intervenção, aumenta ainda a consciencialização sobre a necessidade de mudança de práticas educacionais tradicionais que tendem a desmobilizar as crianças diante das tarefas escolares e, simultaneamente, estabelecer um contato mais próximo e mais sistemático com os professores. ${ }^{(10)}$ Efetivamente, as emoçóes têm um impacte importante na aprendizagem e no rendimento académico dos alunos e as competências emocionais dos professores potenciam esse mesmo impacte. Numa revisão teórica de vários programas para o desenvolvimento de competências sociais e emocionais dos alunos, é explicitamente referido que este tipo de programas diminui problemas de comportamento e enfatizam o valor adaptativo das emoçóes e a importância da regulação emocional tanto nos professores quanto nos alunos. ${ }^{(11)}$

Foi tendo em vista a aplicação futura de um programa de intervenção no âmbito da saúde mental que emergiu o questionamento sobre qual seria o nível de identificação e diferenciação emocional de um grupo de crianças e jovens do ensino básico, definindo-se assim como objetivo central deste estudo: avaliar a competência de alunos do $2^{\circ}$ e $3^{\circ}$ ciclo do Ensino Básico na identificação e diferenciação de emoçóes e sentimentos a partir de situaçóes quotidianas.

\section{Métodos}

Estudo exploratório de corte transversal, desenvolvido no âmbito do Projeto MaiSaúdeMental com referência CENTRO-01-0145-FEDER-023293, desenvolvido na Escola Superior de Saúde do Instituto Politécnico de Viseu, Portugal. Foi realizado com uma amostra de conveniência constituí- 
da por 101 crianças/adolescentes 58,4\% meninas, com idades compreendidas entre os 9 e 15 anos (média = 11,96 anos; $\mathrm{Dp}=1,73$ ), alunos de um Agrupamento Escolar da região, parceiro no Projeto MaiSaúdeMental. Foi utilizado um questionário de caracterização sociodemográfica e o Inventário de Identificação de Emoçôes e Sentimentos (IIES). ${ }^{(1)}$ Este é um inventário de auto-relato que avalia a capacidade das crianças/adolescentes identificarem e diferenciarem emoçóes e sentimentos, partindo de situaçôes quotidianas. É composto por 15 itens que correspondem a 15 situaçóes. Em algumas das situaçôes é fácil perceber o que as crianças/jovens estâo a sentir (por exemplo, se estão a sentir emoçóes ou sentimentos agradáveis ou desagradáveis) e em algumas situaçóes, percebemos o que estão a sentir a partir de sinais que nos dão (por exemplo: O João encontrou o seu cáo que estava perdido. Então, pegou nele ao colo, abraçou-o e deu-lhe beijinhos), noutras situaçôes, não é visível o que o personagem está a sentir, por exemplo: $O$ Paulo está a escrever uma história.

As respostas ao questionário são dicotómicas e abertas ("Há sentimento?" "Sim" ou "Não"; "Qual?") e distribuem-se por 3 escalas: a) Valência Negativa - itens que descrevem situaçóes normalmente associadas à experiência de emoções de tonalidade negativa. A soma dos itens permite obter o Índice de Diferenciação Emocional Negativa; b) Valência Positiva - itens que descrevem situações normalmente associadas à experiência de emoçóes positivas. A soma dos itens permite obter o Índice de Diferenciação Emocional Positiva. No caso dos itens em que está explícito o que os personagens estão a sentir, a partir dos sinais apresentados (Escalas de Valência Positiva e Valência Negativa), as respostas podem assumir valores entre 0 (Zero) e 4 (Quatro) com a seguinte cotação: 0 (Zero) pontos quando a criança não assinala qualquer emoção/ sentimento; entre 1 (um) e 4 (quatro), consoante nas respostas assinalam uma, duas, três ou quatro emoçôes/sentimentos congruentes com a situação apresentada. A soma destas duas escalas permite ainda obter o Índice de Diferenciação Emocional Total. c) Valência Neutra - itens de situaçôes não associadas a qualquer tipo de experiência emocio- nal. Para o caso dos itens em que não está explícito o que os personagens estão a sentir, a partir dos sinais apresentados, a cotação deve ser realizada da seguinte forma: Zero pontos quando a criança assinala que existem emoçóes/sentimentos; um ponto quando a criança refere que não existe emoçôes/ sentimentos. As escalas de valência positiva e negativa e o total da escala de valência neutra permitem avaliar o Índice de Identificação Emocional das crianças.

A aplicação do questionário decorreu em sala de aula, após uma breve explicação do seu funcionamento e esclarecimento de dúvidas. A salvaguarda dos requisitos éticos foi assegurada mediante submissão do protocolo do estudo à Comissão de Proteção de dados (Refa 03.01, 38790 de 18/12/2017), à Direção Geral de Educação (inquérito n.o 0012100017) e após a sua aprovação, foi solicitada autorização ao Conselho Diretivo do Agrupamento de Escolas e obtido o consentimento informado dos Pais/Encarregados de Educação. No tratamento dos dados foi utilizado o programa informático Statistical Package for the Social Sciences ${ }^{\oplus}$ (SPSS versão 24.0) e efetuada análise descritiva e analítica, considerando-se para análise dos testes o Intervalo de Confiança (IC) a 95\% (significância $\mathrm{p}<0,05)$.

\section{Resultados}

As crianças/adolescentes (58,4\% meninas) tinham na sua maioria 10 e 11 anos (24,8 e 18,8\% respetivamente), seguidas das de 13 anos $(15,8 \%)$ e frequentavam maioritariamente o $8^{\circ}$ e $5^{\circ}$ ano de escolaridade (27,7 e 22,8\%) (Tabela 1).

Pela análise dos valores médios das diversas escalas verificou-se que as crianças/adolescentes fazem pouca diferenciação de emoçôes, evidenciando contudo maior capacidade de diferenciação emocional positiva, numa média de 5,11 pontos $(\mathrm{DP}=2,06)$ e um valor médio de diferenciação emocional total de 9,45 pontos $(\mathrm{DP}=2,97)$, com coeficientes de variação $(\mathrm{CV})$ elevados em torno da média $(40,31 \%$ e $31,42 \%$ respetivamente). O Índice de Identificação Emocional médio $(13,36$ pontos; $\mathrm{DP}=3,95)$ indica uma fraca capacidade de identificação de emoçóes, considerando o score máximo da escala $($ score máximo= 39) $($ Tabela 2). 
Tabela 1. Distribuição dos alunos do ensino médio segundo dados sociodemográficos e escolaridade

\begin{tabular}{lcc}
\hline Variávies & & $\mathrm{n}(\%)$ \\
\hline Idade & 9 & $3(3,0)$ \\
& 10 & $25(24,8)$ \\
& 11 & $19(18,8)$ \\
& 12 & $12(11,9)$ \\
& 13 & $18(17,8)$ \\
& 14 & $16(15,8)$ \\
Género & 15 & $8(7,9)$ \\
& Masculino & $42(41,6)$ \\
Total & Feminino & $59(58,4)$ \\
& & $101(100,0)$ \\
Ano escolaridade & & \\
& 4 & $13(12,9)$ \\
& 5 & $23(22,8)$ \\
& 6 & $16(15,8)$ \\
& 7 & $12(11,9)$ \\
& 8 & $28(27,7)$ \\
\hline
\end{tabular}

Tabela 2. Estatísticas dos itens de identificação de emoções e sentimentos dos alunos do ensino básico

\begin{tabular}{lccccc}
\hline Itens & Média & DP & Minimo & Máximo & CV (\%) \\
\hline Diferenciação Emocional Positiva & 5,11 & 2,06 & 2,00 & 13,00 & 40,31 \\
Diferenciação Emocional Negativa & 4,33 & 1,36 & 3,00 & 9,00 & 31,40 \\
Diferenciação Emocional Neutra & 3,91 & 2,17 & 0,00 & 7,00 & 55,49 \\
Diferenciação Emocional Total & 9,45 & 2,97 & 6,00 & 20,00 & 31,42 \\
Índice Identificação Emocional & 13,36 & 3,95 & 7,00 & 26,00 & 29,56 \\
\hline
\end{tabular}

CV - Coeficientes de variação; DP - Desvio-padrão

Pela análise dos valores obtidos em relação ao género, apurou-se pelo teste de U Mann-Whitney que as ordenaçóes médias são mais elevadas no sexo feminino em todos os índices e significativos na Diferenciação Emocional Positiva, (OM= 956.500; p=0,031), Diferenciação Emocional Neutra $(\mathrm{OM}=878.500 ; \mathrm{p}=0,012)$, Diferenciação Emocional Total $(\mathrm{OM}=934.000 ; \mathrm{p}=0,030)$ e no Índice de Identificação Emocional (OM= 692.500; $\mathrm{p}=0,000)$. $\mathrm{Na}$ análise da relação da identificação de emoçóes e sentimentos com a idade e tal como indica o teste de Kruskal-Wallis, os valores foram mais elevados em todas as dimensóes para os alunos de 14 anos e significativas na Diferenciação Emocional Positiva $\left(X^{2}=21,569 ; p=0,001\right)$, na Diferenciação Emocional Total $\left(X^{2}=19,674 ; p=0,003\right)$ e no Índice de Identificação Emocional $\left(X^{2}=17,904 ; p=\right.$ 0,006). Para verificar a influência da escolaridade na capacidade de identificar emoçóes e sentimentos foi realizado o teste de Kruskal-Wallis, apurandose que as médias são mais elevadas em todas as dimensóes para os alunos do $8^{\circ}$ ano, nomeadamente na Diferenciação Emocional Positiva $\left(X^{2}=18,429\right.$, $\mathrm{p}=0,002)$, no Índice de Diferenciação Emocional Total $\left(\mathrm{X}^{2}=17,451 ; \mathrm{p}=0,004\right)$, exceto no Índice de Diferenciação Emocional Negativa que revelou valores médios mais elevados nos alunos do $7^{\circ}$ ano $\left(\mathrm{X}^{2}=11,776, \mathrm{p}=0,038\right)$. Pelos resultados obtidos verificou-se que o reconhecimento das emoçóes aumenta com a idade, é maior no sexo feminino e são mais facilmente identificadas emoçôes positivas do que negativas e/ou neutras.

\section{Discussão}

Os resultados encontrados indicam que as crianças/ adolescentes têm uma fraca capacidade de diferenciar e identificar emoçôes e sentimentos, nomeadamente os negativos e neutros, o que poderá ter efeito negativo no desenvolvimento das diversas tarefas relacionadas com as suas transições desenvolvimentais, com implicaçôes na sua relação com os seus pares e a nível de todo o seu contexto relacional, tal como defendido em diversos estudos. ${ }^{(1,5-7)} \mathrm{A}$ capacidade de reconhecer o estado emocional ou a possibilidade de experienciar múltiplas emoçôes, de identificar as emoçóes dos outros e utilizar vocabulário e expressão de emoçôes de acordo com o nível de desenvolvimento, nomeadamente as emoçôes negativas, tem sido associada a uma mais adequada gestão das próprias emoçôes e das relações socioemocionais nos diversos contextos. Considerando que os problemas de saúde mental constituem um dos principais problemas de saúde pública da sociedade atual a nível mundial ${ }^{(12)}$ reconhece-se a promoção da saúde neste âmbito um dos mais importantes desafios. Neste sentido, a Organização Mundial de Saúde ${ }^{(12)}$ considera o empoderamento socioemocional como uma das fundamentais estratégias a desenvolver a nível familiar e escolar, dada a sua relação custo/benefício estar sobejamente comprovada e reconhecida, tal como indica o estudo de intervenção designado "Atitudes Positivas". ${ }^{(9)}$ Após este programa de intervenção, as crianças do grupo em estudo apresentavam ganhos significativos em três das cinco competências emocionais, especificamente na consciência social e autocontrole, bem como 
diminuição dos níveis de ansiedade. Tal como é reconhecido $^{(11)}$ as crianças que aprendem desde cedo a gerir as suas emoçóes de forma eficaz, conseguem melhores resultados escolares quando comparadas a crianças com capacidades intelectuais semelhantes, mas com défice na sua formação emocional.

No nosso estudo foi ainda apurado que o sexo feminino se distingue dos seus pares do sexo oposto na diferenciação de emoçóes positivas, negativas e neutras e no índice de identificação emocional. Outros autores $^{(8,11,13)}$ sugerem que o sexo feminino expressa mais facilmente os seus sentimentos, sendo também mais hábil na utilização das palavras em substituição de determinadas reaçóes emocionais. Uma vez que os rapazes, culturalmente são menos incitados a verbalizar os seus sentimentos e emoçóes, tendencialmente tornam-se menos conscientes dos estados emocionais, tanto próprios como dos outros indivíduos. Na sequência destes resultados, existe alguma discordância afirmando-se que enquanto persista uma educação diferenciada entre os géneros, dificilmente se comprovará a ideia de que o sexo feminino lida e manifesta melhor, de uma forma inata, as emoçóes. ${ }^{(14)}$ Estas evidências ligadas às diferenças de gênero na expressão de emoções em contextos específicos têm implicações importantes no desenvolvimento saudável (mesmo que diferenciado face ao género) e mal adaptativo das crianças. ${ }^{(8)}$

Num estudo realizado sobre inteligência emocional e comportamentos disruptivos em alunos da educação básica ${ }^{(15)}$ concluiu-se igualmente diferenças relativas ao género, apurando-se que os rapazes apresentavam mais comportamentos disruptivos do que as meninas e que existe uma relação significativa entre os comportamentos disruptivos e os índices gerais de inteligência emocional, habilidade intimamente relacionada com as competências socioemocionais.

Considerando-se ainda a evolução cronológica da idade e consequentemente da escolaridade, verificou-se que a identificação de emoçóes e sentimentos era mais elevada nos alunos mais velhos, aumentando de forma gradual com a idade, mas apenas significativa na diferenciação emocional positiva. Outros estudos $^{(8)}$ demonstram que a inteligência emocional e social se diferencia com a idade e que esta diferen- ça persiste em associação com o género, indicando as meninas mais emoçôes positivas e emoçôes internalizantes (ie: tristeza, ansiedade, simpatia) que os meninos, sendo estas diferenças mais pronunciadas com o aumento da idade, distinguindo-se ainda o tipo de emoçóes. Neste caso os meninos revelaram mais emoçôes externalizantes que as meninas na idade pré-escolar e menos na adolescência.

Face aos resultados apurados, e tendo em conta as evidências científicas, considera-se muito importante que a identificação das emoçóes e a capacitação emocional seja uma habilidade iniciada desde idades precoces, seguindo programas estruturados e ministrados na escola e ao longo de toda a escolaridade obrigatória. Estes devem seguir uma lógica de formaçáo preventiva com foco na empatia e na comunicação não-violenta, que terão decerto impacto na diminuição de fenómenos como o bullying e outros comportamentos, tanto externalizantes quanto internalizantes, que condicionam a saúde e o sucesso escolar e comprometem a saúde emocional e mental.

Apesar dos resultados, o estudo revela algumas limitações, sobretudo associadas ao seu caráter transversal, às características não aleatórias da amostra e ao baixo número de participantes.

\section{Conclusão}

As principais conclusóes indicam que as crianças e adolescentes têm fraca capacidade de identificação e diferenciação emocional, evidenciando contudo maior capacidade na diferenciação de emoçóes positivas. Verificou-se ainda maior competência de identificação e diferenciação emocional no género feminino e que estas competências aumentam com a idade.

\section{Agradecimentos}

O nosso agradecimento ao Fundo Social Europeu, ao Programa Operacional CENTRO 2020 e à Fundação para a Ciência e Tecnologia do Governo Português, instituições públicas que se consti- 
tuem como fontes de financiamento do Projeto de Investigação MAISaúde Mental (código de identificação: CENTRO-01-0145-FEDER-023293), onde este estudo se integra.

\section{Colaborações}

Aparício G, Ferreira M, Campos S, Ribeiro O, Amaral O, Cruz C, Duarte J e Martins R declaram que contribuíram com a concepção, análise e interpretação de dados, elaboração do artigo, revisão crítica do conteúdo e aprovação final da versão a ser publicada.

\section{Referências}

1. Moreira PA, Oliveira JT, Crusellas L, Lima A. Inventário de Identificação de Emoções e Sentimentos (IIES): estudo de desenvolvimento e de validação. Rev Psicol Criança Adolesc. 2012;3(1):39-66.

2. Moreira PA, Dias PC, Pettrachi P. Características psicométricas do questionário de envolvimento entre pais e professores. Rev Psicol Criança Adolesc. 2012;3(1):123-35.

3. Israelashvili J, Oosterwijk S, Sauter D, Fischer A. Knowing me, knowing you: emotion differentiation in oneself is associated with recognition of others' emotions. Cogn Emotion. 2019;33(7):1461-71.

4. Erbas Y, Ceulemans E, Lee Pe M, Koval P, Kuppens P. Negative emotion differentiation: its personality and well-being correlates and a comparison of different assessment methods. Cogn Emotion. 2014;28(7):1196-213.
5. Emery NN, Simons JS, Clarke CJ, Gaher RM. Emotion differentiation and alcohol-related problems: the mediating role of urgency. Addict Behav. 2014;39(10):1459-63.

6. Zaki LF, Coifman KG, Rafaeli E, Berenson KR, Downey G. Emotion differentiation as a protective factor against nonsuicidal self-injury in borderline personality disorder. Behav Ther. 2013;44(3):529-40.

7. Erbas Y, Sels L, Ceulemans E, Kuppens P. Feeling Me, Feeling You: the relation between emotion differentiation and empathic accuracy. Soc Psychol Personal Sci. 2016;7(3):240-7.

8. Chaplin TM, Aldao A. Gender differences in emotion expression in children: a meta-analytic review. Psychol Bull. 2013;139(4):735-65.

9. Coelho V, Sousa V, Raimundo R, Figueira A. The impact of a Portuguese middle school social-emotional learning program. Health Promot Int. 2017;32(2):292-300.

10. Ferreira M, Duarte J, Campos S, Chaves C, Felizardo S, Cardoso AP. Emotional Skills and Promotion of School Success: Preliminary Data of an Intervention Programme with Parents. Eur Proc Social Behav Sci. 2017;30:310-7.

11. Durlak JA, Weissberg RP, Dymnicki $A B$, Taylor RD, Schellinger KB. The impact of enhancing students' social and emotional learning: a meta-analysis of school-based universal interventions. Child Dev. 2011;82(1):405-32.

12. World Health Organization. Prevention of mental disorders: Effective interventions and policy option. Geneva: World Health Organization; [Internet] 2004 [cited 2019 Mar 13]. Available from: https://www.who. int/whr/2004/en/

13. Brody LR, Hall JA. Gender and emotion in context. In: Lewis M, Haviland-Jones JM, Barrett LF, editors. Handbook of emotions. 3rd ed. New York: The Guilford Press; 2008. pp. 395-408.

14. Muller-Lissniner A. A inteligência emocional na criança: Como estimulá-la no seu filho. Cascais: Pergaminho; 2001.

15. Esturgó-Deu ME, Sala-Roca J. Disruptive behaviour of students in primary education and emotional intelligence. Teach Teach Educ. 2010;26(4):830-7. 